\title{
Tratamiento del bruxismo del sueño en niños.
}

\section{Resumen}

Los enfoques actuales para el tratamiento del Bruxismo del Sueño en niños se focalizan en el manejo de los síntomas y la prevención de complicaciones, siendo muy amplio el espectro de alternativas, para utilizar en forma individual o combinada. Se recomienda identificar y controlar los factores causales asocia-
María Laura Hermida, ${ }^{1}$ Silvina Gabriela Cortese, ${ }^{2}$ Sandra Kalil Bussadori, ${ }^{3}$

Roxana Ferreira, ${ }^{4}$

Lorena Spatakis, ${ }^{5}$ Asociación Latinoamericana de Odontopediatría.

Artigo da revisao

\section{Tratamento do bruxismo do sono em crianças.}

\section{Resumo}

As abordagens atuais para o tratamento do bruxismo do sono em crianças se concentram no manejo dos sintomas e na prevenção de complicações, sendo o espectro de alternativas muito amplo, a ser utilizado individualmente ou em dos al bruxismo. Las posibilidades de tratamiento varían desde la educación del paciente y la familia, el uso da aparatos intraorales, y las técnicas psicológicas hasta la medicación. Teniendo en cuenta lo complejo del tema se hace necesario un abordaje multidisciplinario para el tratamiento del Bruxismo del Sueño en niños.

Palabras clave: Bruxismo del Sueño, tratamiento, niños.

1. Directora del Departamento de Odontología, Universidad Católica del Uruguay, Montevideo, Uruguay.

2. Profesora Asociada. Facultad de Odontología, Cátedra Odontología Integral Niños, Universidad de Buenos Aires, Buenos Aires, Argentina.

3. Profesora del curso de Especialización en Odontopediatría APCD/EAP. Profesora del programa de Maestría en Ciencias de Rehabilitación UNINOVE/ SP, Brasil.

4. Instructora Especialidad de Ortodoncia y Ortopedia Bucomaxilofacial, Universidad Católica del Uruguay.

5. Odontopediatra Servicio Médico Integral, Montevideo, Uruguay. 
uma abordagem multidisciplinar para o tratamento do bruxismo do sono em crianças.
Palavras chave: Bruxismo do Sono, tratamento, crianças.

Article of revision

\section{Treatment of sleep bruxism in children.}

\begin{abstract}
Current approaches for Sleep Bruxism treatment in children focus on symptom management and prevention of related complications. A broad spectrum of alternatives is identified that can be used individually or in combination. It is recommended to identify and control the causal factors associated with sleep
\end{abstract}

\section{Introducción}

El bruxismo ha sido definido como una actividad repetida de la musculatura masticatoria, caracterizada por apretar o rechinar los dientes y/o apretar o impulsar la mandíbula. Presenta dos manifestaciones: bruxismo en vigilia (BV) y bruxismo del sueño (BS), respondiendo a dos expresiones circadianas distintas, dependiendo del momento de ocurrencia de la actividad. ${ }^{1}$ Esta definición fue avalada por la Asociación Americana de Medicina del Sueño. ${ }^{2}$

La regulación central está considerada como factor etiológico del BS vinculado con la neurotransmisión de dopamina. ${ }^{3,4}$ Sin embargo la literatura ha sido enfocada a los signos y síntomas, tanto para el diagnóstico como para el tratamiento del BS. $^{5}$ bruxism. Treatment possibilities range from patient and family education, orthopedic appliances, psychological techniques up to medication. Taking into account the complexity of this topic, a multidisciplinary approach is needed for Sleep Bruxism management in children.

Key words: Sleep Bruxism, treatment, children.

La clasificación más reciente y actualmente aceptada para el BS, tipifica el BS en posible, probable y definitivo., ${ }^{1,4}$ Los métodos diagnósticos actuales en los que se basa la clasificación no incluyen medidas centrales de bruxismo del sueño (sistema catecolaminérgico), aún cuando ya se ha determinado que su etiología es central. ${ }^{3,4}$

Un reporte previo recopiló los aspectos generales relacionados con el Bruxismo, recogidos durante el Simposio sobre Bruxismo en Niños realizado en San Pablo en mayo de 2015 y la Mesa Latinoamericana sobre Bruxismo infantil, que tuvo lugar durante el XVIII Congreso de la Asociación Latinoamericana de Odontopediatría, llevado a cabo en Santa Marta, Colombia en Abril de 2016. ${ }^{5}$ Como conclusión se resaltó la importancia de la participación del odontopediatra en 
equipos multidisciplinarios para poder ofrecer el mejor tratamiento y resultado para sus pacientes. Reconociendo la relevancia del tema, se continuó realizando profundización y trabajo colaborativo entre todas las sociedades de Odontopediatría miembros de ALOP, siendo expuestas las conclusiones en el desarrollo de la Mesa Latinoamericana Tratamiento del Bruxismo Infantil durante el XIX Congreso de la Asociación Latinoamericana de Odontopediatría y I Congreso de la Sociedad Uruguaya de Odontopediatría, realizado en Montevideo, Uruguay, en 2018.

A continuación se presenta la recopilación de las diferentes ponencias, basadas en la literatura y compartidas por los colegas integrantes de la Mesa.

\section{Tratamiento}

Considerando la regulación central del bruxismo, los múltiples factores concomitantes, y las diferentes manifestaciones clínicas: apretamiento, rechinamiento, propulsión mandibular, diurno y/o nocturno, es indiscutible que cada paciente debe ser individualmente evaluado y tratado. La severidad de los síntomas será dependiente de la duración, la frecuencia y la intensidad. Los enfoques actuales se focalizan en el manejo de los síntomas y la prevención de complicaciones, siendo muy amplio el espectro de alternativas, para utilizar en forma individual o combinada. ${ }^{6}$

Un consenso de expertos que tuvo lugar en San Francisco en Marzo de 2017 revisó los aspectos generales del bruxismo, y recomendó que los estudios futuros deben estar dirigidos a una mejor comprensión de los posibles correlatos clínicos, tanto negativos como positivos, dentro del paradigma del bruxismo como un comportamiento en individuos sanos. Concluyeron que el desafío será establecer el enfoque más confiable y válido (individual o combinados) que también sea el más factible. ${ }^{4}$

Se ha mencionado la importancia de identificar y controlar los factores causales asociados al bruxismo. Teniendo en cuenta que el bruxismo puede ser secundario a algunos factores tales como obstrucción de vías aéreas o SAOS, la identificación de las causas ambientales y su control es esencial en el abordaje clínico del paciente con bruxismo. ${ }^{7}$

La Academia Americana de Odontología Pediátrica (AAPD) expresa que existe evidencia de bruxismo infantil autolimitante, y que el espectro del tratamiento varía desde la educación del paciente y la familia, el uso de aparatos intraorales, y las técnicas psicológicas hasta la medicación. ${ }^{2}$

La educación del paciente y la familia se orienta a establecer rutinas de sueño y control de otros hábitos orales diurnos tales como onicofagia, masticación de objetos y chicle. También la terapia miofuncional colabora para concientizar y automatizar patrones de masticación, deglución y posición de reposo sin contacto dentario. Las terapias psicológicas pueden brindar herramientas para el control de los factores emocionales de autoexigencia, inestabilidad emocional y ansiedad. ${ }^{8}$

Basado en la evidencia científica disponible, los ajustes oclusales no están indicados en el abordaje clínico 
del bruxismo, dado que esa condición es de etiología central y no periférica. El entendimiento actual sobre la etiopatogenia del bruxismo no avala ese procedimiento clínico como correcto. ${ }^{3,5}$

Los enfoques actuales para el tratamiento del BS se focalizan en el manejo de los síntomas y la prevención de complicaciones. En los casos en los que hay desgaste oclusal severo se ha reportado el uso de las coronas de acero para rehabilitar la función masticatoria y eliminar la posible sintomatología dolorosa ocasionada por pérdida de estructura dentaria. Los pacientes con Trastornos del Espectro Autista (TEA) presentan una variedad de síntomas médicos y del comportamiento, entre los cuales se menciona el bruxismo del sueño. En los casos de pacientes portadores de TEA que presentan severo desgaste dentario, el tratamiento podría incluir el uso de coronas de acero inoxidable para restaurar el tejido coronario perdido. 9,10,11

En cuanto al uso de aparatos intraorales, si bien pueden proteger del desgaste dentario y relajar los músculos de la masticación, en casos de bruxismo del sueño, no hay evidencia de su efectividad para resolver el bruxismo. Sin embargo, los aparatos bimaxilares de ortopedia funcional en forma indirecta evitaría el apretamiento, el rechinamiento y la propulsión mandibular. ${ }^{8}$ En pacientes con Bruxismo del Sueño asociado a Síndrome de Apnea Obstructiva del Sueño (SAOS), los aparatos intraorales protegen del desgaste dentario y relajan los músculos de la masticación. ${ }^{8}$ Algunos reportes mencionan el uso de dispositivos intraorales para avance mandibular (MOA) en población infantil con SAOS, concluyendo que estos dispositivos pueden ser considerados como una alternativa para su tratamiento. ${ }^{12}$

Las placas oclusales han sido bien documentadas en la literatura, siendo el tratamiento de elección de los odontólogos. Hay pocos estudios en niños. Restrepo et al., en un ensayo clínico controlado, en niños, utilizaron la placa oclusal rígida para el tratamiento del bruxismo y concluyeron que la utilización de la misma no fue eficiente en la reducción de los síntomas de bruxismo. Sin embargo, a pesar de no influir en los parámetros fisiológicos de la condición, la placa es eficiente para el tratamiento de trastornos temporomandibulares en dentición permanente completa. ${ }^{13}$

En la literatura se menciona que los niños con bruxismo muestran una posición de la cabeza significativamente adelantada, que sugiere una relación entre bruxismo y postura cervical. Una placa rígida oclusal (biteplate) es una forma común de tratamiento del bruxismo. En estos casos el propósito de esta placa sería estabilizar y estimular la función de la articulación temporomandibular y los músculos de la masticación, disminuir la actividad muscular anormal y proteger los dientes de una excesiva fricción y cargas traumáticas. Además, las placas pueden ser utililzadas para promover una posición estable $\mathrm{y}$ funcional de la articulación y una oclusión ideal, que a su vez reorganice la actividad neuromuscular. ${ }^{14} \mathrm{El}$ uso de las placas en la dentición primaria debe ser restringido, estando indicado sólo cuando el desgaste puede ocasionar daño biológico para el niño, lo que es poco frecuente. En la dentición mixta, está contraindicado por completo, debido a la posible restricción del crecimiento de los arcos. El control y seguimiento es siempre importante. ${ }^{5,8}$ 
Algunos autores han sugerido que la fisioterapia puede brindar beneficios en el tratamiento del bruxismo. Dentro de las técnicas fisioterapéuticas más utilizadas se encuentra la estimulación neuromuscular transcutánea (TENS), estimulación neural eléctrica por microcorriente (MENS), terapia de enfriamiento (crioterapia), ultrasonido, terapia de infrarrojos, cinesioterapia, masoterapia y láser. ${ }^{6,8,15}$

Dentro del plan de tratamiento para pacientes con bruxismo del sueño, se menciona la necesidad de reducir la tensión física, muscular y el manejo de los aspectos psicológicos asociados, tratando los signos y síntomas para lograr el rompimiento del patrón neuromuscular habitual. Por presentar etiología compleja se hace necesario un enfoque multiprofesional, comprendiendo la utilización en conjunto o aislada de fisioterapia, farmacoterapia, terapias odontológicas y terapia psicológica. ${ }^{8,16,17}$ Como el bruxismo frecuentemente se relaciona con un componente psicológico, la evaluación de niveles de estrés y ansiedad parece ser un modo importante de obtener información en torno a ese disturbio, así como sobre las terapias utilizadas para el control del mismo. Un método que se ha mostrado eficaz para medir el estrés y la ansiedad es la cuantificación del cortisol en la saliva. ${ }^{18}$ Se sabe que la ansiedad y el estrés estimulan la secreción de esa hormona por la glándula suprarrenal. ${ }^{18-19}$ Sin embargo, Cortese y col (2019) no encontraron diferencias significativas. En su estudio observaron que el cortisol aumenta en estrés agudo, mientras que en adaptación recupera valores normales. $^{20}$ Mc Cartan et al (1996), encontraron correlación positiva entre ansiedad y la concentración de cortisol salivar, siendo este un método confiable y no invasivo que determina situaciones estresantes. En este sentido, este análisis puede determinar situaciones estresantes independientemente del origen ser psicológico, físico o ambiental. ${ }^{21-23}$

Los agentes farmacológicos se han usado para tratar el bruxismo del sueño focalizados enlos sistemas neuroquímicos implicados en la actividad motora orofacial. A pesar del uso experimental de una variedad de compuestos, no hay enfoques farmacológicos ampliamente aceptados para el tratamiento del bruxismo. Por otra parte estos estudios fueron dirigidos a niños con compromiso sistémico. ${ }^{24}$

Los resultados de un ensayo clínico que utilizó diazepam no respaldan su uso para el manejo del BS en niños sanos, debido al escaso beneficio obtenido $y$ presencia de eventos adversos. Se sugiere que el uso de benzodiazepinas tales como diazepam podría ser más efectivo en combinación con otras modalidades de tratamiento para el tratamiento del BS, pero esto debería ser evaluado en estudios posteriores. ${ }^{25,26}$

La medicina homeopática ha sugerido la utilización de Melissa officinalis (MO), Phytolacca decandra (PD) y una combinación de ambas para el tratamiento del bruxismo. Melissa officinalis L ha sido empleada como una terapia natural debido a las propiedades sedativas, ansiolíticas, anti inflamatorias y antiespasmódicas de los aceites esenciales que la componen.

Un estudio llevado a cabo utilizando ambas sustancias demostró resultados promisorios con el uso de $\mathrm{MO}$ en el 
tratamiento del "posible" bruxismo del sueño en niños, pero no así cuando ambas sustancias se utilizaron combinadas. ${ }^{27} \mathrm{En}$ otro ensayo clínico no se encontraron diferencias estadísticamente significativas entre la actividad muscular inicial y final en la comparación en cada grupo y entre grupos. Los autores concluyeron que el uso de Melissa officinalis L en la dosis utilizada no condujo a reducción de la actividad muscular en niños con bruxismo. ${ }^{28}$

Una revisión sistemática y meta-análisis reciente reporta que la evidencia sobre tratamiento del BS en niños es limitada. Los autores afirman que son necesarios futuros estudios con diseño apropiado, conducidos en un número adecuado de pacientes que aseguren la validez de los mismos y que estén basados en criterios diagnósticos estandarizados. Dentro de las conclusiones mencionan que la terapia con hidroxicina mostró una gran eficacia en el BS, mientras que las terapias con Melissa officinalis presentaron bajos grados de asociación con disminución de síntomas de BS. ${ }^{29}$

\section{Conclusiones}

En función de lo expresado por los diferentes profesionales latinoamericanos convocados puede consensuarse la necesidad del abordaje multidisciplinario basado en un diagnóstico integral. A la fecha la evidencia científica y los niveles de recomendación para el tratamiento del bruxismo en niños, aún son muy escasos. Si bien hay un amplio espectro de posibilidades propuestas, el énfasis está puesto en identificar y controlar los factores causales. Ante la falta de evidencia y la controversia en relación al tema es necesario el trabajo mancomunado de los referentes e investigadores latinoamericanos.

\section{Notas del artículo}

Mesa Latinoamericana ALOP: "Tratamiento del Bruxismo Infantil". Participantes: Silvina Cortese (Argentina), Carla Rodríguez (Puerto Rico), Milton Marquiegui (Bolivia), Roxana Ferreira (Uruguay), Lorena Spatakis (Uruguay). Coordinadores: Paulo Redua (Brasil), Laura Hermida (Uruguay).

\section{Referencias bibliográficas}

1. Lobbezoo F, Ahlberg J, Glaros AG, Kato T, Koyano K, Lavigne GJ, de Leeuw R, Manfredini D, Svensson P, Winocur E. Bruxism defined and graded: an international consensus. J Oral Rehabil., 2013; 40:2-4

2. American Academy of Sleep Medicine. International classification of sleep disorders (ICDS)-, 3rd ed. Darien, IL: American Academy of Sleep Medicine, 2014.

3. Lobbezoo F, Naeije M. Bruxism is mainly regulated centrally, not peripherally. J Oral Rehabil. 2001; 28: 1085-1091

4. Lobbezoo F, Ahlberg J, Raphael KG, Wetselaar P, Glaros AG et al. International consensus on the assessment of bruxism: Report of a work in progress. J Oral Rehabil. 2018 November ; 45(11): 837844. doi:10.1111/joor.12663.

5. Hermida Bruno L, Restrepo Serna C, ALOP. Bruxismo del sueño y Sindrome de Apnea Obstructiva del Sueño en Niños. Revisión narrativa. 2016; 6 (2): 99-107.

6. Guitelman I, Fridman D, Savia A, Ienco M, Mondello A, Farah C, Biondi A, Cortese S. Conocimiento de padres y cuidadores sobre consecuencias de bruxismo y parafunciones. XLIX Reunión anual de la Sociedad Argentina de Investigación Odontológica (SAIO). Mar del Plata, Buenos Aires, 
Argentina. 2016. Disponible en: http://www.saio.org.ar/new/descargas/Libro_XLIX_Reunion_ Cientifica_Anual.pdf

7. Kobayashi FY, Gavião MBD, Marquezin MCS, Fonseca FLA, Montes ABM, Barbosa TS, Castelo PM. Salivary stress biomarkers and anxiety symptoms in children with and without temporomandibular disorders. Braz Oral Res. 2017; 31:e78.

8. Asociación Latinoamericana de Odontopediatría. XIX Congreso Latinoamericano de Odontopediatría (conferencias). 18 al 20 de octubre 2018; Montevideo, Uruguay

9. Muthu MS, Prathibha KM. Management of a child with autism and severe bruxism: a case report. J Indian Soc Pedod Prev Dent 2008; 26(2):82-4.

10. Friedlander AH, Yagiela JA, Paterno VI, Mahler ME. The pathophysiology, medical management, and dental implications of autism. J Calif Dent Assoc 2003; 31: 681-2.

11. Chew LC, King NM, O.Donnell D. Autism: The etiology, management and implications for treatment modalities from the dental perspective. Dent Update 2006;33:70-2,74-6,78-80

12. Modesti-Vedolin G, Chies C, Chaves-Fagondes S, Piza-Pelizzer E, Lima- Grossi M. Efficacy of a mandibular advancement intraoral appliance (MOA) for the treatment of obstructive sleep apnea syndrome (OSAS) in pediatric patients: A pilot-study. Med Oral Patol Oral Cir Bucal. 2018 1;23 (6):e656-63.

13. Restrepo CC, Medina I, Patiño I. Effect of occlusal splints on the temporomandibular disorders, dental wear and anxiety of bruxist children. Eur J Dent. 2011;5: 441-450

14. Bortoletto CC, Cordeiro da Silva F, Silva PF, Leal de Godoy CH, Albertini R, Motta LJ, MesquitaFerrari RA, Fernandes KP, Romano R, Bussadori SK. Evaluation of Cranio-cervical Posture in Children with Bruxism Before and After Bite Plate Therapy: A Pilot Project. J Phys Ther Sci. 2014; 26(7):1125-8.

15. Kato T, Montplaisir JY, Guitard F, Sessle BJ, Lund JP, Lavigne GJ. Evidence that experimentally induce sleep bruxism is a consequence of transientarousal. J Dent Res. 2003; 82:284-8.

16. Raphael KG, Marbach JJ, Klausnerr JJ, Teaford MF. Is bruxism severity a predictor of oral splint efficacy in patients with myofascial face pain? J Oral Rehabil. 2003;30(1):17-29.

17. Serra Negra JM, Paiva SM, Fulgencio LB et al. Enviromental factors,sleep duration, and sleep bruxism in Brazilian schoolchilldren:a case control study Sleep Med. 2014 Feb; 2014(2):236-9.

18. Amanto JN, Tuon RA, Castelo PM, Gavião MB, Barbosa T de S. Assessment of sleep bruxism, orthodontic treatment need, orofacial dysfunctions and salivary biomarkers in asthmatic children. Arch Oral Biol. 2015May;60(5):698-705. doi:10.1016/j archoralbio. 2015.02.011.Epub 2015

19. Cruz T, Falci S, Galvao E. Association Between Bruxism and Salivary Cortisol Levels: A Systematic Review. Int. J. Odontostomat 2016; 10: 3: 469-474

20. Silvina Gabriela Cortese, Ingrid Clarisa Guitelman, Ana María Biondi. Cortisol salival en niños con y sin bruxismo. Rev Odontoped Latinoam. 2019; 9 (1). Disponible en https://www. revistaodontopediatria.org/ediciones/2019/1/art-3/

21. Santos MJP, Bernabé DG, Nakamune ACMS, Perri SHV, Aguiar SMHCA, Oliveira SHP. Salivary alpha amylase and cortisol levels in children with global developmental delay and their relation with the expectation of dental care and behavior during the intervention. Res Develop Disabil., 2012; 33(2):499-505.

22. Manfredini D, Restrepo C, Diaz-Serrano K, Winocur E, Lobbezoo F. Prevalence of sleep bruxism in children: A systematic review of the literature. J Oral Rehabil. 2013;40:631-342.

23. Silva ML, Mallozi MC, Ferrari GF. Salivary cortisol to assess the hypothalamic-pituitaryadrenal axis in healthy children under 3 years old. J Pediatr. 2007; 83(2):121-6.

24. Scrivani SJ, Khawaja SN, Bavia PF. Nonsurgical Management of Pediatric Temporomandibular Joint Dysfunction. Oral Maxillofac Surg Clin North Am. 2018 Feb;30(1):35-45

25. Seyyed-Nassereddin Mostafavi, Azadeh Jafari, Shervin Ghaffari Hoseini, Majid Khademian, Roya Kelishadi. The efficacy of low and moderate dosage of diazepam on sleep bruxism in children: A randomized placebo-controlled clinical trial. J Res Med Sci. 2019; 24: 8.

26. Lal SJ, Weber KK. Bruxism Management. StatPearls Publishing; 2018 Jan-. Disponible en: https:// www.ncbi.nlm.nih.gov/books/NBK482466/

27. Tavares-Silva C, Holandino C, Homsani F, Luiz RR, Prodestino J, Farah A, Lima JP, Simas RC, Castilho CVV, Leitão SG, Maia LC, Fonseca-Gonçalves A. Homeopathic medicine of Melissa officinalis combined or not with Phytolacca decandra in the treatment of possible sleep bruxism in children: A 
crossover randomized triple-blinded controlled clinical trial. Phytomedicine. 2019; 58:152869. doi: 10.1016/j.phymed.2019.152869.

28. Carolina Carvalho Bortoletto, Fernanda Cordeiro da Silva, Monica da Consolação Canuto Salgueiro, Lara Jansiski Motta, Lucia Maria Curiki, Raquel Agnelli Mesquita-Ferarri, Kristianne Porta Santos Fernandes, Sandra Kalil Bussadori. Evaluation of electromyographic signals in children with bruxism before and after therapy with Melissa Officinalis L - a randomized controlled clinical trial. J. Phys. Ther. Sci. 2016; 28: 738-742.

29. Ierardo G, Mazur M, Luzzi V, Calcagnile F, Ottolenghi L, Polimeni A. Treatments of sleep bruxism in children: A systematic review and meta-analysis. Cranio. 2019; 26:1-7. doi: 10.1080/08869634.2019.1581470.

Recibido: $16 / 10 / 19$

Aceptado: 31/10/19

Correspondencia: Laura Hermida, e-mail: mlaura.hermida@ucu.edu.uy 